\title{
Mortalidad y comorbilidades perioperatorias en pacientes con amputaciones disvasculares
}

\section{Perioperative morbidity and mortality in patients with dysvascular amputations}

\author{
Francisco Regalado-Solís ${ }^{1}$, Abraham Ziga-Martínez², Sandra Olivares-Cruz ${ }^{1}$, Miguel Á. Sierra-Juárez ${ }^{1}$, \\ Enrique Santillán-Aguayo ${ }^{1}$, Wenceslao Fabián-Mijangos ${ }^{1}$ y Verónica Carbajal-Robles ${ }^{1 *}$ \\ ${ }^{1}$ Servicio de Angiología y Cirugía Vascular, Unidad 503, Hospital General de México "Dr. Eduardo Liceaga"; "Servicio de Angiología y Cirugía \\ Vascular, Hospital Ángeles Mocel. Ciudad de México, México
}

\begin{abstract}
Resumen
Antecedentes: Las amputaciones disvasculares son un tratamiento común para los pacientes en los que otras medidas han fracasado. Objetivo: Evaluar la mortalidad a 30 días y al año posterior a una amputación disvascular. Material y métodos: Estudio retrospectivo descriptivo, con 127 pacientes evaluados desde el 1 de marzo del 2018 al 28 de febrero del 2019, se examinaron los patrones de morbilidad y mortalidad secundario a amputaciones disvasculares de extremidades inferiores. Resultados: Se evaluaron 132 extremidades amputadas asociadas a isquemia, evidenciando mortalidad a 30 días de 20.47\% y al año de $50.39 \%$. El riesgo de mortalidad a un año en pacientes sometidos a amputación por arriba de la rodilla con HR 1.974 (IC: 1.407-2.331, $p=0.049$ ). Conclusión: Los resultados coinciden con la literatura, en que las amputaciones disvasculares se asocian a mortalidad significativa, con un incremento de la mortalidad hasta del $70 \%$ en pacientes con enfermedad renal crónica y/o cardiopatía isquémica.
\end{abstract}

Palabras clave: Insuficiencia arterial aguda. Insuficiencia arterial crónica. Amputación disvascular. Amputación mayor. Mortalidad.

\begin{abstract}
Background: Amputations remains as a common treatment for patients in whom other measures have failed. Objective: To evaluate the outcomes at 30 days and one year after major disvascular amputation. Material and methods: Descriptive, retrospective study, using data from 127 consecutive patients from March 1, 2018, to February 28, 2019. We examined morbidity and mortality patterns after major lower extremity disvascular amputations. Results: 132 major amputations were evaluated, associated with ischemia, showing 30 -day mortality of $20.47 \%$ and an overall mortality at one year of $50.39 \%$. One-year mortality risk in patients undergoing above the knee amputation was HR 1.974 (Cl: 1.407-2.331, $p=0.049$ ). Conclusion: The outcomes are similar with the ones described in modern literature in that disvascular amputations were associated with significant mortality in the follow-up and rising to $70 \%$ in end stage renal disease and ischemic heart disease patients.
\end{abstract}

Key words: Acute arterial disease. Chronic arterial disease. Disvascular amputation. Major amputation. Mortality.

\section{Correspondencia:}

*Verónica Carbajal-Robles

E-mail: veronica_carbajal85@ hotmail.com

0377-474010 2021 Sociedad Mexicana de Angiol bajo la licencia CC BY-NC-ND license (http://creativecommons.org/licenses/by-nc-nd/4.0/).
Fecha de recepción: 14-02-2021

Fecha de aceptación: 02-08-21

DOI: 10.24875/RMA.21000009
Disponible en internet: 24-09-2021 Rev Mex Angiol. 2021;49(3):80-87 www.RMAngiologia.com
s un artículo open access 


\section{Introducción}

La prevalencia de la enfermedad arterial periférica (EAP) en la población mundial se estima entre el 3 y el $5 \%$ y en población diabética del 10 al $20 \%$, y es la tercera causa de morbilidad cardiovascular aterosclerótica, después de la enfermedad coronaria y la enfermedad cerebrovascular². Esta patología suele ser asintomática hasta en un $50 \%$ de los casos; pero en aproximadamente el $4 \%$ de pacientes no diabéticos y el $12 \%$ de pacientes diabéticos se manifiesta clínicamente como isquemia arterial de extremidades inferiores ${ }^{1-3}$. En México, el alto índice de obesidad es un indicador indirecto en la prevalencia de patologías cronicodegenerativas como diabetes mellitus (DM), hipertensión arterial sistémica (HAS), enfermedad renal crónica (ERC) y EAP. La incidencia de EAP es del $7.66 \%$ en pacientes mayores de 50 años y del $17.8 \%$ en mayores de 70 años, con mayor prevalencia para el sexo masculino y pacientes fumadores persistentes ${ }^{4-8}$.

El $90 \%$ de las amputaciones mayores de extremidades inferiores son atribuidas a la presencia de enfermedades crónico-degenerativas; de estas, el $60 \%$ son secundarias a procesos infecciosos, el $30 \%$ a insuficiencia arterial y el restante $10 \%$ a trauma, cáncer y ciertos padecimientos congénitos ${ }^{9-11}$. Los procesos isquémicos son una causa importante de amputaciones, ya que como se mencionó previamente son responsables del $30 \%$ de todos los casos. Solamente en el 2015, EE.UU. reportó 800,000 casos de amputaciones disvasculares $(A D)$, con una tasa de mortalidad superior al $50 \%$ tras el primer año del procedimiento ${ }^{4,5}$.

El estado clínico vascular previo a una amputación mayor debe ser determinado por medio de escalas validadas como las descritas por Rutherford ${ }^{12}$, o más recientemente la publicada por la Society of Vascular Surgery, la escala Wound, Ischemia, and foot Infection classification system (WIfl) ${ }^{13}$, que destacan a la insuficiencia arterial aguda como la isquemia súbita debida a trombosis, embolias o cuadros agudizados producto de cirugías de salvamento y/o revascularización no exitosas que llegan a producir cambios isquémicos irreversibles de las extremidades, y en sus manifestaciones tardías de EAP como cuadro crónico secundario a cambios de conformación de la pared vascular, limitación en su remodelación y/o disminución del diámetro interno del vaso por cambios ateroescleróticos ${ }^{14}$. Debido a esto, la intervención por especialistas calificados es vital, ya que una intervención inicial adecuada o incluso una amputación primaria oportuna disminuye cuadros de síndrome de reperfusión, rabdomiólisis y/o insuficiencia renal aguda, o en casos de pacientes no recuperables y/o crónicos se disminuye la incidencia de síndromes coronarios, eventos cerebrales vasculares (EVC) o exacerbación de cuadros de insuficiencia cardiaca ${ }^{15-17}$, en ambos grupos se han reportado tasas de mortalidad asociadas elevadas ${ }^{18}$.

La mortalidad se encuentra directamente relacionada con procedimientos de revascularización no exitosos, factores de riesgo cardiovascular y niveles más proximales de amputación. La sobrevida después de AD mayor por debajo de la rodilla se encuentra entre el 65 y el $80 \%$, con disminución considerable en amputaciones por arriba de la rodilla, llegando al $50 \%$ tras el primer año de la amputación ${ }^{19-21}$, por lo que el objetivo primario del estudio fue determinar la sobrevida perioperatoria y a un año tras la realización de AD mayores.

\section{Material y métodos}

Estudio longitudinal, descriptivo, análisis retrospectivo que comprendió el periodo del 1 de enero del 2018 al 28 de febrero del 2019 y donde se evaluaron los expedientes de 127 pacientes en seguimiento por consulta externa en el servicio de angiología y cirugía vascular con diagnóstico amputación de extremidad/es inferior/es secundaria a insuficiencia arterial aguda 0 crónica. El estudio fue aprobado por el comité de investigación del centro hospitalario bajo el dictamen con número de registro DECS/JPO-CT-337-2020. Se tomaron en cuenta todos los expedientes que cumplieran los criterios de ingreso descritos más adelante.

\section{Criterios de selección: inclusión, exclusión y eliminación}

Se incluyeron todos los expedientes consecutivos de pacientes mayores a 18 años, y a quienes se les hubiera realizado amputación de extremidad inferior durante el periodo de la cohorte, definidas según su tipo en primarias (debido a deterioro tisular y funcional extenso, insuficiencia arterial irreversible) y secundarias, siendo estas posteriores a procedimientos vasculares de urgencia/electivos no exitosos, tanto quirúrgicos abiertos (p. ej., derivaciones femoropoplíteas, trombectomías o endarterectomías femorales) como endovasculares (p. ej., angioplastias, colocación de endoprótesis vasculares, trombólisis fármaco-mecánicas) que culminaron en amputación mayor de la extremidad tratada. De aquí se definió como AD mayor a toda amputación (supracondílea o infracondílea) por un mal estado de perfusión de la extremidad con deterioro tisular, funcional y/o neurológico, resultando en la no viabilidad de 
esta. Se incluyó dentro de la definición de insuficiencia arterial a todo paciente con EAP sintomática que hubiera sido evaluado y con mediciones de índice tobillo brazo menores a 0.9 y/o que en su registro de evaluación vascular estuviera incluido el diagnóstico de clínico de «insuficiencia arterial» valorado por medio de escalas validadas (Rutherford, WIfl) o métodos diagnósticos como angiotomografía y ultrasonido Doppler; se tomó en cuenta además a pacientes con diagnóstico clínico de «pie diabético», definido para fines del estudio como un proceso infeccioso, ulceración y/o pérdida de tejido de la extremidad en estudio, y se incluyeron en este rubro únicamente a los que además de este diagnóstico hubieran sido evaluados y asociados al proceso infeccioso y se documentara afección vascular por los mismos métodos previamente descritos. Se definió revascularización no exitosa a todo intento de salvamento de extremidad que, a pesar de haber logrado éxito técnico, culminó en la pérdida de esta, secundaria a isquemia, o exacerbación de cuadros de infección y/o dolor. Se excluyó a todo paciente cuyo diagnóstico de amputación fue diferente a etiología vascular, datos del expediente clínico y/o electrónico incompletos (que faltaran datos esenciales como tipo de amputación, notas de seguimiento perioperatorio y/o más del $15 \%$ de datos en la hoja de recolección de datos) o que la hoja de valoración de cirugía vascular no describiera el diagnóstico de EAP y/o insuficiencia arterial, pacientes no tratados en nuestro centro hospitalario y/o que no contaran con valoración vascular previa al procedimiento quirúrgico en estudio; además se eliminaron los expedientes de pacientes no localizados al momento del seguimiento, de pacientes que no desearon participar en el estudio y de pacientes con causa de mortalidad diferente a variables de riesgo cardiovascular descritas (p. ej., cáncer, homicidio, traumatismos, etc.).

\section{Procedimiento}

Se realizó la revisión de las listados de consulta externa e interconsultas hospitalarias y de urgencias buscando como denominador común pacientes que en el momento de la valoración inicial ameritaron amputación indistintamente de su causa, en un segundo tiempo se realizó revisión sistemática de expedientes clínicos y electrónicos de pacientes y se identificó exclusivamente pacientes que cursaron con $A D$ mayor, bajo los criterios de inclusión y no inclusión ya descritos, seleccionando únicamente expedientes completos con hoja de valoración vascular previa al tratamiento quirúrgico, registrándole en el instrumento de recolección de datos, el cual se dividió en características demográficas (edad, sexo), antecedentes, tabaquismo, apartado de examen físico donde se documentó el estadio clínico (Rutherford y/o WIfl), los datos relacionados a tipo de amputación, fecha de esta, reintervenciones y complicaciones si las hubieron. Para el apartado de seguimiento y la valoración del objetivo principal de sobrevida se realizó la revisión de datos en el expediente acerca de registro de citas posteriores a la cirugía y datos relacionados con la defunción, y para los pacientes en los cuales no se encontró esta información se recabaron los datos del contacto en el programa de admisión hospitalaria, hoja de trabajo social y/o del expediente clínico para comprobación de supervivencia vía telefónica.

\section{Análisis estadístico}

Se recabó en la base de datos y toda la información se vació en el programa SPSS v.25 (IBM, IL, EE.UU.), donde se describieron las características generales de la población estudiada mediante estadística descriptiva con medidas de tendencia central y dispersión de variables cuantitativas y con frecuencias simples en números absolutos y relativos para variables categóricas. Se utilizaron pruebas estadísticas paramétricas y no paramétricas, para comparar grupos según el nivel de amputación, y modelo multivariante de Cox para predictores de mortalidad. Se realizó estimación de la sobrevida utilizando análisis de Kaplan-Meier y se tomó un valor de $p<0.05$ como estadísticamente significativo.

\section{Resultados}

Las características básicas de la población del estudio se muestran en la tabla 1. La edad media de los pacientes fue de $65 \pm 15$ años y 79 pacientes $(62 \%)$ eran hombres; DM presente en 86 pacientes $(68 \%)$, HAS en 71 pacientes (56\%) y ERC en 19 pacientes (15\%). Como factor de riesgo prevalente se registró tabaquismo en el $50 \%$ de los pacientes y un $12 \%$ más que ya había abandonado el hábito al momento de la evaluación inicial. En la tabla 1 también se describen las características clínicas de los pacientes en su valoración inicial, según su presentación clínica con la escala de Rutherford, describiendo como lesiones extensas al $32.57 \%$, con lesiones distales al antepié al $23.48 \%$ (ambas con o sin procesos infecciosos asociados), y la tercera presentación clínica más común fueron los pacientes que consultaron tardíamente por cuadros irreversibles de 
insuficiencia arterial, con el $21.96 \%$, no encontrando pacientes crónicos con claudicación intermitente.

\section{Antecedentes médicos}

Del total de expedientes examinados se reportaron 86 pacientes $(67.71 \%)$ con $\mathrm{DM}, 71$ pacientes $(55.90 \%)$ con HAS, 19 pacientes (14.96\%) con ERC, 8 pacientes (6.30\%) con antecedente de EVC, 10 pacientes $(7.87 \%)$ con cardiopatía isquémica y 8 pacientes $(6.29 \%)$ como sanos (Tabla 1).

\section{Características de la amputación actual}

Ciento veintisiete pacientes con 132 extremidades inferiores amputadas (122 unilaterales y 5 bilaterales), $31(24.40 \%)$ amputaciones por debajo de la rodilla y $96(75.60 \%)$ por arriba de la rodilla, descritas en la tabla 1; 89 extremidades se amputaron de manera primaria y 43 de manera secundaria a revascularización no exitosa, procesos infecciosos derivados de dichos procedimientos o dolor neuropático tras la revascularización. De estas 132 intervenciones, se llevaron a cabo 35 reintervenciones (26.51\%), 9 para elevación del nivel de amputación, 15 procedimientos de lavado/drenaje absceso/cierre diferido y/o colocación de sistema de presión negativa y 11 desarticulaciones, de las cuales fallecieron 7 por cuadros de sepsis de manera temprana; además, 34 extremidades $(25.75 \%)$ cursaron con complicaciones menores que se manejaron ambulatoriamente, 18 casos con infección local, 5 pacientes (3.78\%) cursaron con seromas realizando drenaje local y manejo de herida abierta, 5 pacientes $(3.78 \%)$ con trombosis venosas con manejo con anticoagulación y compresión del muñón, 3 pacientes $(2.27 \%)$ cursaron con necrosis cutánea en el borde quirúrgico, tratado con manejo avanzado de heridas, y 3 pacientes (2.27\%) refirieron neuropatía/parestesias asociándose con síndrome de dolor fantasma y enviándoles a seguimiento por clínica del dolor y servicio de rehabilitación.

Con respecto a las causas de amputación, 29 extremidades $(21.97 \%)$ por insuficiencia arterial aguda irreversible, $31(23.48 \%)$ tras la revascularización no exitosa de extremidades, $23(17.42 \%)$ por lesiones isquémicas extensas proximales al antepié, $34(25.76 \%)$ por asociación a procesos infecciosos con involucro del tobillo, 9 pacientes $(6.82 \%)$ con amputaciones previas contralaterales no rehabilitados y/o en abandono social clasificados como no funcionales, y 6 (4.54\%) por dolor tras la revascularización, estos últimos definidos también como revascularizaciones no exitosas, pero se mencionan de esta manera por ser una causa poco estudiada de amputación que será objeto de subanálisis posterior (Tabla 1).

\section{Mortalidad}

La mortalidad global del estudio fue del $50.39 \%$ (Fig. 1), 35 (44.30\%) hombres y $29(60.41 \%)$ mujeres (Tabla 2). La mortalidad global en pacientes fumadores fue del $50 \%$, con mayor afectación en mujeres, del $58.33 \%$. De acuerdo con el nivel de amputación (Fig. 1C), falleció el $25.8 \%$ de los pacientes con amputaciones realizadas por debajo de la rodilla y el $58.33 \%$ de las amputaciones realizadas por arriba de esta.

La tasa de mortalidad global asociada a comorbilidades fue del $45.35 \%$ en DM, el $52.11 \%$ en HAS y el $68.42 \%$ en ERC (Fig. 2); de estos últimos 7 fallecieron tempranamente, 4 por falla multiorgánica, 3 por causas relacionadas a su enfermedad, el $37.5 \%$ asociado a EVC, el $70 \%$ en cardiopatía isquémica y el $3.93 \%$ con fibrilación/arritmias, de los cuales en su totalidad fueron mujeres, 2 de las cuales fallecieron durante su internamiento y 3 antes de cumplir 6 meses tras la amputación. Con respecto a la mortalidad a 30 días, se registraron 26 pacientes fallecidos antes de los 30 días de la intervención (5 amputaciones infracondíleas y 21 supracondíleas), de los cuales 21 fallecieron durante su internamiento y 5 pacientes egresaron y de los cuales 2 fallecieron por falla multiorgánica (altas voluntarias) y 3 egresaron, falleciendo por causas relacionadas a insuficiencia renal; de los 21 fallecimientos durante el internamiento, se registró como la causa más común de deceso el choque séptico y falla multiorgánica. Con respecto a la mortalidad acumulada a un año, fueron 64 pacientes (50.39\%), y como primera causa de esta el síndrome coronario isquémico (14 pacientes), EVC (11 pacientes), eventos relacionados con ERC 12 y 11 pacientes por causas que se desconocen.

\section{Discusión}

Este estudio examinó las tasas de mortalidad por todas las causas entre los pacientes que se sometieron a $A D$ tanto por abajo como por arriba de la rodilla, dándoles seguimiento hasta por 22 meses. En el análisis univariante y multivariante de Cox el riesgo de mortalidad en general fue 1.4 veces más alto en personas con antecedentes de ERC (intervalo de confianza del 95\% [IC 95\%]: 1.192-1.831; $p=0.014$ ) que en el resto de los pacientes y en personas con antecedentes de cardiopatía isquémica fue de casi dos veces (hazard ratio [HR]: 1.98; IC 95\%: 1,6699-2.3581; $p=0.03$ ), 
Tabla 1. Características basales de los pacientes

\begin{tabular}{|c|c|c|c|}
\hline Variable, valor* & Global & Sexo masculino & Sexo femenino \\
\hline N. ${ }^{0}$ pacientes & 127 & $79(62)$ & $48(38)$ \\
\hline N. ${ }^{0}$ extremidades amputadas & 132 & $81(62)$ & $41(38)$ \\
\hline $\begin{array}{l}\text { Localización } \\
\text { Derecha } \\
\text { Izquierda } \\
\text { Bilateral }\end{array}$ & $\begin{array}{l}65(51) \\
57(45) \\
5(4)\end{array}$ & $\begin{array}{l}44(33) \\
33(25) \\
2(3)\end{array}$ & $\begin{array}{l}21(16) \\
24(18) \\
3(4)\end{array}$ \\
\hline $\begin{array}{l}\text { Características de los pacientes } \\
\text { Edad media (años) +/- DE } \\
>\text { de } 65 \text { años }\end{array}$ & $\begin{array}{l}65 \pm 15 \\
65(51)\end{array}$ & $\begin{array}{c}62 \\
35(54)\end{array}$ & $\begin{array}{c}69 \\
30(46)\end{array}$ \\
\hline $\begin{array}{l}\text { Sexo } \\
\text { Femenino } \\
\text { Masculino }\end{array}$ & $\begin{array}{l}48(38) \\
79(62)\end{array}$ & & \\
\hline $\begin{array}{l}\text { Antecedentes } \\
\text { Tabaquismo } \\
\text { Fumador actual } \\
\text { No fumador } \\
\text { Exfumador }\end{array}$ & $\begin{array}{l}63(50) \\
49(38) \\
15(12)\end{array}$ & $\begin{array}{l}48(76) \\
25(51) \\
6(40)\end{array}$ & $\begin{array}{c}15(24) \\
24(49) \\
9(60)\end{array}$ \\
\hline Diabetes mellitus & $86(68)$ & $55(64)$ & $31(36)$ \\
\hline Hipertensión arterial sistémica & $71(56)$ & $42(59)$ & $29(41)$ \\
\hline Enfermedad renal crónica & $19(15)$ & $16(84)$ & $3(16)$ \\
\hline Historia de enfermedad coronaria isquémica & $10(8)$ & $8(80)$ & $2(20)$ \\
\hline Historia de enfermedad cerebral vascular & $8(6)$ & $4(50)$ & $4(50)$ \\
\hline Enfermedad pulmonar obstructiva crónica & $7(5)$ & $3(43)$ & $4(57)$ \\
\hline Sin antecedentes & $8(6)$ & $5(62)$ & $3(38)$ \\
\hline Características de la amputación actual & & & \\
\hline $\begin{array}{l}\text { Tipo de amputación } \\
\text { Primaria } \\
\text { WIfl estadio } 5 / \text { infeccioso } \\
\text { Isquemia irreversible } \\
\text { Lesiones isquémicas extensas } \\
\text { Paciente no funcional }\end{array}$ & $\begin{aligned} 28(21) \\
29(22) \\
23(17) \\
9(7)\end{aligned}$ & $\begin{array}{l}23(82) \\
15(52) \\
15(65) \\
4(44)\end{array}$ & $\begin{array}{l}5(18) \\
14(48) \\
8(35) \\
5(56)\end{array}$ \\
\hline $\begin{array}{l}\text { Secundaria } \\
\text { Tratamiento quirúrgico } \\
\text { Tratamiento endovascular } \\
\text { Infección de tejidos blandos posterior a tratamiento quirúrgico } \\
\text { Dolor neuropático tras revascularización }\end{array}$ & $\begin{aligned} & 19(14) \\
& 12(9) \\
& 6(5) \\
& 6(5) \\
& 132(100)\end{aligned}$ & $\begin{array}{l}7(37) \\
9(75) \\
5(83) \\
3(50) \\
81(61)\end{array}$ & $\begin{array}{l}12(63) \\
3(25) \\
1(17) \\
3(50) \\
51(39)\end{array}$ \\
\hline $\begin{array}{l}\text { Presentación clínica } \\
\text { Agudo } \\
\text { Rutherford IIa } \\
\text { Rutherford IIb } \\
\text { Rutherford III }\end{array}$ & $\begin{array}{l}3(2) \\
14(11) \\
29(22)\end{array}$ & $\begin{array}{c}1(33) \\
9(64) \\
15(52)\end{array}$ & $\begin{array}{c}2(66) \\
5(36) \\
14(48)\end{array}$ \\
\hline $\begin{array}{l}\text { Crónico } \\
\text { Rutherford } 4 \\
\text { Rutherford } 5 \\
\text { Rutherford } 6\end{array}$ & $\begin{array}{c}12(9) \\
31(23) \\
43(33) \\
132(100)\end{array}$ & $\begin{array}{l}10(83) \\
18(58) \\
28(65) \\
81(61)\end{array}$ & $\begin{array}{l}2(17) \\
13(42) \\
15(35) \\
51(39)\end{array}$ \\
\hline $\begin{array}{l}\text { Nivel de amputación } \\
\text { Infracondílea } \\
\text { Supracondílea }\end{array}$ & $\begin{array}{l}31(24) \\
96(76)\end{array}$ & $\begin{array}{l}24(77) \\
55(57)\end{array}$ & $\begin{array}{c}7(23) \\
41(43)\end{array}$ \\
\hline $\begin{array}{l}\text { Reintervención } \\
\text { Elevación del nivel de amputación } \\
\text { Desarticulación } \\
\text { Lavado/drenaje absceso/cierre diferido }\end{array}$ & $\begin{array}{c}9(7) \\
11(9) \\
15(12)\end{array}$ & $\begin{array}{l}4(44) \\
4(36) \\
8(53)\end{array}$ & $\begin{array}{l}5(56) \\
7(64) \\
7(47)\end{array}$ \\
\hline $\begin{array}{l}\text { Complicaciones menores } \\
\text { Infección local } \\
\text { Dehiscencia herida } \\
\text { Trombosis venosa } \\
\text { Necrosis cutánea } \\
\text { Dolor fantasma } \\
\text { sin complicaciones }\end{array}$ & $\begin{aligned} & 18(14) \\
& 5(4) \\
& 5(4) \\
& 3(2) \\
& 3(2) \\
& 63(48)\end{aligned}$ & $\begin{array}{c}12(67) \\
3(60) \\
3(60) \\
3(100) \\
2(67) \\
42(67)\end{array}$ & $\begin{array}{c}6(33) \\
2(40) \\
2(40) \\
0(0) \\
1(33) \\
21(33)\end{array}$ \\
\hline
\end{tabular}

* Los valores entre paréntesis son porcentajes. 


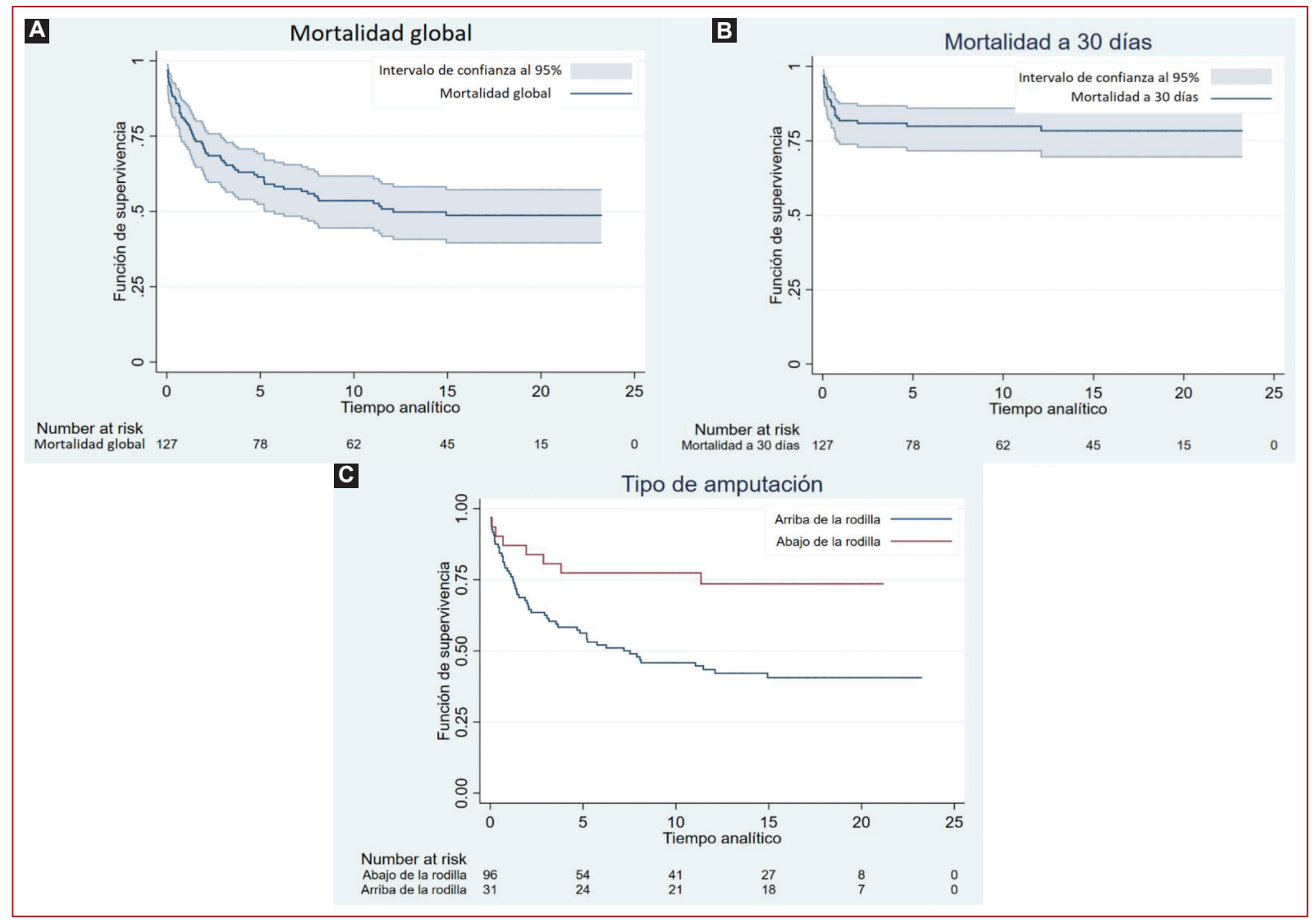

Figura 1. Mortalidad global y según tipo de amputación. Análisis de supervivencia de Kaplan-Meier en la cohorte total de 127 pacientes. A: 64 pacientes fallecen durante el seguimiento. B: 26 pacientes fallecen durante los primeros 30 días de seguimiento. C: estratificado por tipo de procedimiento (amputaciones por debajo y por arriba de la rodilla).

Tabla 2. Mortalidad

\begin{tabular}{|c|c|c|c|c|c|c|}
\hline Mortalidad general & $n=127$ & $(\%)$ & 30 días & $(\%)$ & 1 año & $(\%)$ \\
\hline $\begin{array}{l}\text { Falleció } \\
\text { Sexo masculino } \\
\text { Sexo femenino }\end{array}$ & $\begin{array}{l}64 \\
35 \\
29\end{array}$ & $\begin{array}{l}50.39 \\
54.68 \\
45.31\end{array}$ & $\begin{array}{c}26(21)^{*} \\
14(9)^{*} \\
12(12)^{*}\end{array}$ & $\begin{array}{c}20.47(16.53)^{*} \\
11.02(7.08)^{*} \\
9.45(9.45)^{*}\end{array}$ & $\begin{array}{l}38 \\
21 \\
17\end{array}$ & $\begin{array}{l}29.92 \\
16.54 \\
13.32\end{array}$ \\
\hline No falleció & 63 & 49.61 & & & & \\
\hline Total & 127 & 100 & & & & \\
\hline $\begin{array}{l}\text { Causa de mortalidad } \\
\text { Síndrome coronario isquémico } \\
\text { Insuficiencia cardiaca } \\
\text { Evento cerebral vascular } \\
\text { Insuficiencia renal } \\
\text { Falla multiorgánica } \\
\text { Sepsis } \\
\text { Arritmias } \\
\text { Se desconoce }\end{array}$ & $\begin{array}{c}14 \\
4 \\
11 \\
12 \\
2 \\
7 \\
3 \\
11\end{array}$ & $\begin{array}{c}21.88 \\
6.25 \\
17.19 \\
18.75 \\
3.13 \\
10.94 \\
4.69 \\
17.19\end{array}$ & $\begin{array}{l}2(1)^{*} \\
2(2)^{*} \\
2(2)^{*} \\
7(5)^{*} \\
2(2)^{*} \\
6(6)^{*} \\
0(0)^{*} \\
5(3)^{*}\end{array}$ & $\begin{array}{c}7.69(3.85)^{*} \\
7.69(7.69)^{*} \\
7.69(7.69)^{*} \\
26.92(19.23)^{*} \\
7.69(7.69)^{*} \\
23.08(23.08)^{*} \\
0.00(0.00)^{*} \\
19.53(11.54)^{*}\end{array}$ & $\begin{array}{l}12 \\
2 \\
9 \\
5 \\
0 \\
1 \\
3 \\
6\end{array}$ & $\begin{array}{c}31.58 \\
5.26 \\
23.68 \\
13.16 \\
0.00 \\
2.63 \\
7.89 \\
15.79\end{array}$ \\
\hline Total & 64 & 100.00 & $26(21)^{*}$ & $100(81)^{*}$ & 38 & 100.00 \\
\hline
\end{tabular}

*Evento ocurrido durante estancia hospitalaria; los valores entre paréntesis son porcentajes.

mortalidad al año de amputaciones por arriba de la rodilla, mientras que no se encontró diferencia significativa entre el nivel de amputación y la mortalidad temprana (Tabla 3). 


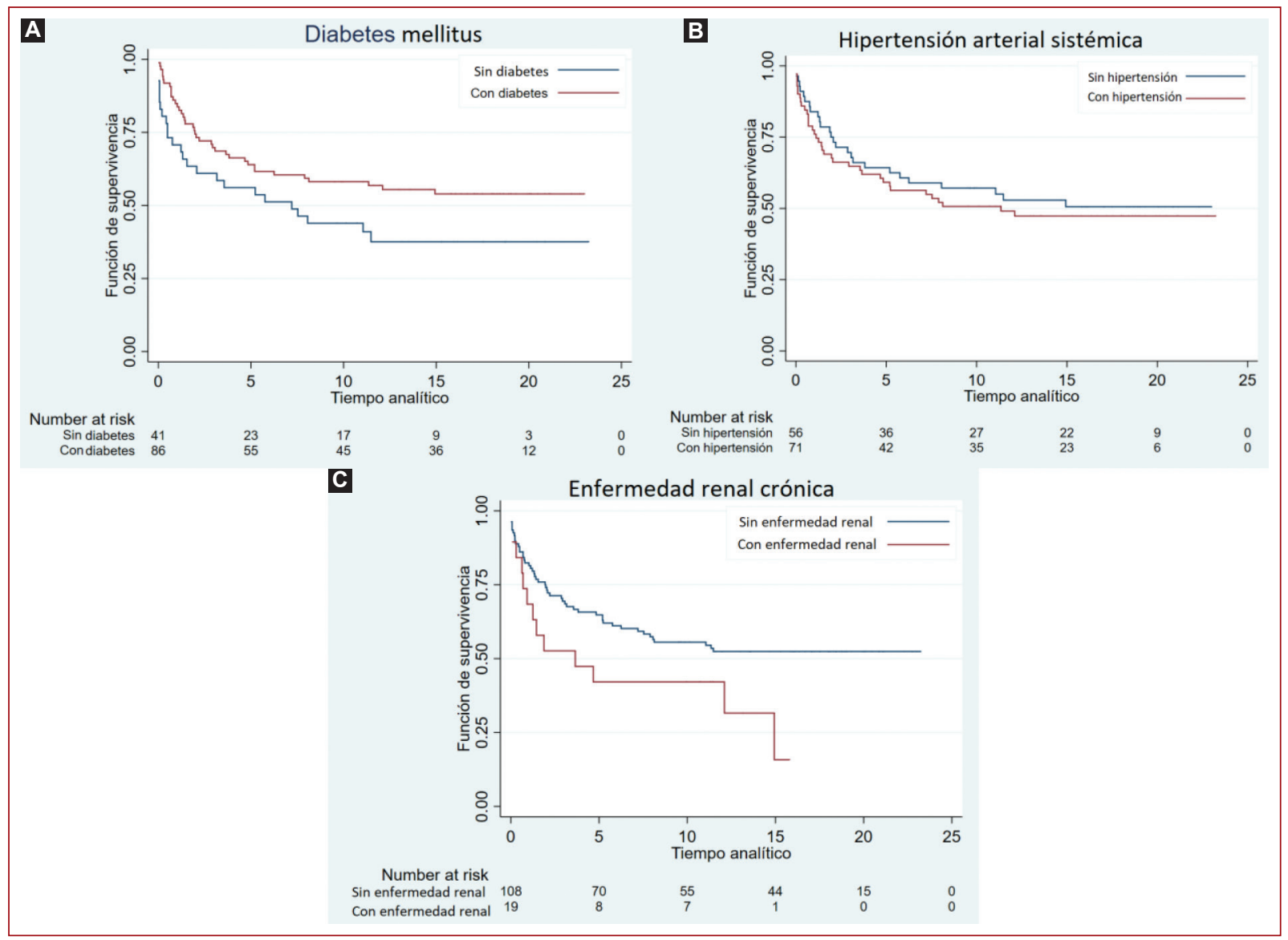

Figura 2. Mortalidad asociada a comorbilidades. Análisis de Kaplan-Meier de la supervivencia asociada a comorbilidades como factores de riesgo clásicos de enfermedad arterial periférica. A: diabetes mellitus, la mortalidad global fue del $45.35 \%$. B: hipertensión arterial sistémica, la tasa global de mortalidad fue del $52.11 \%$. C: enfermedad renal crónica, la tasa global de mortalidad fue del $68.42 \%$.

Tabla 3. Resumen de análisis univariante y multivariante de regresión de Cox de mortalidad

\begin{tabular}{|l|c|c|c|c|c|c|}
\hline \multirow{2}{*}{ Variable } & \multicolumn{3}{|c|}{ Univariante } & \multicolumn{3}{c|}{ Multivariante } \\
\cline { 2 - 8 } & Hazard ratio & (IC 95\%) & $\mathbf{p}$ & Hazard ratio & (IC 95\%) & p \\
\hline Sexo & 1.048 & $(0.178-1.799)$ & 0.879 & 0.747 & $(0.425-1.311)$ & 0.309 \\
\hline Tabaquismo & 0.988 & $(0.571-1.924)$ & 0.969 & 0.680 & $(0.385-1.202)$ & 0.184 \\
\hline Diabetes mellitus & 0.979 & $(0.537-1.817)$ & 0.948 & 1.279 & $(0.693-2.362)$ & 0.431 \\
\hline Hipertensión arterial sistémica & 0.930 & $(0.525-1.826)$ & 0.819 & 0.645 & $(0.368-1.131)$ & 0.126 \\
\hline Enfermedad renal crónica & 1.377 & $(0.500-1.730)$ & 0.011 & 1.399 & $(1.192-1.831)$ & 0.014 \\
\hline Cardiopatía isquémica & - & - & - & 1.981 & $(1.669-2.358)$ & 0.031 \\
\hline Amputación supracondílea & 1.932 & $(1.400-2.173)$ & 0.071 & 1.974 & $(1.407-2.331)$ & 0.049 \\
\hline
\end{tabular}

IC: intervalo de confianza

siendo ambas diferencias estadísticamente significativas. En relación con la mortalidad y el nivel de amputación (HR: 1.974; IC 95\%: 1.407-2.331; $p=0.049$ ), fue estadísticamente significativa únicamente para 
Dentro de las limitaciones del estudio se incluyen los datos recabados de un único centro y el tamaño pequeño de la población estudiada, además de la documentación y registro hospitalario, los cuales no siempre son exactos y/o se encuentran incompletos. No se documentó el efecto de la intervención vascular previa a la amputación ni el posible impacto de estas revascularizaciones exitosas.

\section{Conclusiones}

La tasa de mortalidad reportada refleja la fragilidad de la población del estudio, ya que falleció la mitad de los pacientes en el primer año posterior a la amputación y la mortalidad a 30 días fue superior a los reportes de la literatura. La EAP confiere un alto riesgo cardiovascular, por lo que su diagnóstico y tratamiento oportuno es importante para determinar el pronóstico, no solamente libre de amputación, sino de la sobrevida en general, por lo que centros de tercer nivel y/o referencia nacional deben de establecer algoritmos y protocolos de tratamiento multidisciplinario que incluyan valoración de lesiones no traumáticas de extremidades inferiores por médicos especialistas para diagnosticar y dar tratamiento oportuno a cualquier grado de insuficiencia arterial.

\section{Financiamiento}

La presente investigación no ha recibido ninguna beca específica de agencias de los sectores públicos, comercial o sin ánimo de lucro.

\section{Conflicto de intereses}

Los autores declaran no tener conflicto de intereses.

\section{Responsabilidades éticas}

Protección de personas y animales. Los autores declaran que para esta investigación no se han realizado experimentos en seres humanos ni en animales.

Confidencialidad de los datos. Los autores declaran que han seguido los protocolos de su centro de trabajo sobre la publicación de datos de pacientes.

Derecho a la privacidad y consentimiento informado. Los autores han obtenido el consentimiento informado de los pacientes y/o sujetos referidos en el artículo.

\section{Bibliografía}

1. Boyko EJ, Monteiro-Soares M, Wheeler SGB. Peripheral arterial disease, foot ulcers, lower extremity amputations, and diabetes. En: Cowie CC, Casagrande SS, Menke A, Cissell MA, Eberhardt MS, Meigs JB, et al., editores. Diabetes in America. $3^{\text {rd }}$ edition. Bethesda (MD): National Institute of Diabetes and Digestive and Kidney Diseases (US); agosto 2018. Disponible en: https://www.niddk.nih.gov/about-niddk/strategic-plans-reports/diabetes-in-america-3rd-edition

2. Fowkes FG, Rudan D, Rudan I, Aboyans V, Denenberg JO, McDermott $\mathrm{MM}$, et al. Comparison of global estimates of prevalence and risk factors for peripheral artery disease in 2000 and 2010: A systematic review and analysis. Lancet. 2013;382(9901):1329-40.

3. Persiani $\mathrm{F}$, et al. Complications and mortality after major amputation in patients with critical limb ischemia: A single center experience. J Foot Ankle Res. 2019;7:1-6.

4. Mundell BF, Luetmer MT, Kremers HM, Visscher S, Hoppe KM, Kaufman KR. The risk of major cardiovascular events for adults with transfemoral amputation. J Neuroeng Rehabil. 2018;15(Suppl 1):58.

5. Simons JP, Schanzer A, Flahive JM, Osborne NH, Mills JL Sr Bradbury AW, et al. Survival prediction in patients with chronic limb-threatening ischemia who undergo infrainguinal revascularization. J Vasc Surg. 2019;69(6S):137S-151S.e3.

6. Trujillo-Alcocer JC, Flores-Escartín MH, López-Monterrubio AR, Serrano-Lozano JA. Prevalencia de la enfermedad arterial periférica en población mexicana derechohabiente del Hospital Regional Lic. Adolfo López Mateos, ISSSTE. Rev Mex Angiol. 2014;42(2):62-7.

7. Buitrón-Granados LV, Martínez-López C, Escobedo-de la Peña J. Prevalence of peripheral arterial disease and related risk factors in an urban Mexican population. Angiology. 2004;55(1):43-51.

8. van Netten JJ, Fortington LV, Hinchliffe RJ, Hijmans JM. Early post-operative mortality after major lower limb amputation: A systematic review of population and regional based studies. Eur J Vasc Endovasc Surg. 2016;51(2):248-57.

9. Fard B, Dijkstra PU, NEDA Study Group; Voesten HGJM, Geertzen JHB. Mortality, reamputation, and preoperative comorbidities in patients undergoing dysvascular lower limb amputation. Ann Vasc Surg. 2020;64:228-38.

10. Fashandi AZ, Mehaffey JH, Hawkins RB, Kron IL, Upchurch GR Jr, Robinson WP. Major adverse limb events and major adverse cardiac events after contemporary lower extremity bypass and infrainguinal endovascular intervention in patients with claudication. J Vasc Surg. 2018;68(6):1817-23.

11. Fard B, Dijkstra PU, Stewart RE, Geertzen JHB. Incidence rates of dysvascular lower extremity amputation changes in Northern Netherlands: A comparison of three cohorts of 1991-1992, 2003-2004 and 20122013. PLoS One. 2018;13(9):e0204623.

12. Rutherford RB, Baker JD, Ernst C, Johnston KW, Porter JM, Ahn S, et al. Recommended standards for reports dealing with lower extremity ischemia: revised version. J Vasc Surg. 1997;26(3):517-38. Erratum in: J Vasc Surg. 2001;33(4):805.

13. Mills JL Sr, Conte MS, Armstrong DG, Pomposelli FB, Schanzer A, Sidawy AN, et al.; Society for Vascular Surgery Lower Extremity Guidelines Committee. The Society for Vascular Surgery Lower Extremity Threatened Limb Classification System: risk stratification based on wound, ischemia, and foot infection (WIfI). J Vasc Surg. 2014;59(1):220-34.e1-2.

14. Fitridge R, Pena G, Mills JL. The patient presenting with chronic limb-threatening ischaemia. Does diabetes influence presentation, limb outcomes and survival? Diabetes Metab Res Rev. 2020;36(Suppl 1):e3242.

15. Dillon M, Fatone S, Quigley M. While mortality rates differ after dysvascular partial foot and transtibial amputation, should they influence the choice of amputation level? Arch Phys Med Rehabil. 2017;98(9):1900-2.

16. Roepke AM, Turner AP, Henderson AW, Goldberg SB, Norvell DC, Czerniecki JM, et al. A prospective longitudinal study of trajectories of depressive symptoms after dysvascular amputation. Arch Phys Med Rehabil. 2019;100(8):1426-33.e1.

17. Dillon MP, Quigley M, Fatone S. Outcomes of dysvascular partial foot amputation and how these compare to transtibial amputation: a systematic review for the development of shared decision-making resources. Syst Rev. 2017;6(1):54.

18. Jones WS, Patel MR, Dai D, Vemulapalli S, Subherwal S, Stafford J, et al. High mortality risks after major lower extremity amputation in Medicare patients with peripheral artery disease. Am Heart J. 2013;165(5):809-15.e1.

19. Wied C, Foss NB, Tengberg PT, Holm G, Troelsen A, Kristensen MT. Avoidable 30-day mortality analysis and failure to rescue in dysvascular lower extremity amputees. Acta Orthop. 2018;89(2):246-50.

20. Arhuidese I, Nejim B, Aji EA, Canner J, Malas MB. Survival after major lower extremity amputation in patients with end-stage renal disease. J Vasc Surg. 2019;70(4):1291-8.

21. Ploeg AJ, Lardenoye JW, Vrancken Peeters MP, Breslau PJ. Contemporary series of morbidity and mortality after lower limb amputation. Eur $\mathrm{J}$ Vasc Endovasc Surg. 2005;29(6):633-7 